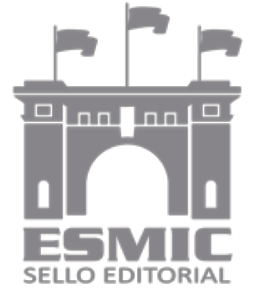

Revista Científica General José María Córdova

ISSN 1900-6586 (impreso), 2500-7645 (en línea)

Volumen 16, Número 21, enero-marzo 2018, pp. 1-16

http://dx.doi.org/10.21830/19006586.311

Citación: Mendoza Pérez, C., y Vitola Quintero, M. (2018, enero-marzo). Herramienta virtual para simular maniobras con buques tipo GNL en la bahía de Cartagena. Rev. Cient. Gen. José María Córdova 16 (21), 1-16. DOI: http://dx.doi.org/10.21830/19006586.311

\title{
Herramienta virtual para simular maniobras con buques tipo GNL en la bahía de Cartagena
}

Sección: Estudios MiLITARes

Artículo de investigación científica y tecnológica

Cristhian Carmelo Mendoza Pérez ${ }^{\mathrm{a}}$

Escuela Naval de Cadetes "Almirante Padilla", Cartagena, Colombia

Marena del Rosario Vitola Quintero ${ }^{\mathrm{b}}$

Escuela Naval de Cadetes "Almirante Padilla", Cartagena, Colombia

A virtual tool to simulate maneuvering of LNG ships in the Cartagena bay

Uma ferramenta virtual para simular manobras com navios de GNL na baía de Cartagena

Outil virtuel pour simuler des manœuvres avec types de navires LNG dans la baie de Carthagene

Recibido: 30 de junio de 2017 • Aceptado: 23 de noviembre de 2017

a https://orcid.org/ 0000-0001-5219-3615 - Contacto: poseidon@enap.edu.co

b https://orcid.org/ 0000-0002-7885-815X - Autor de correspondencia - Contacto: marena.vitola@armada.mil.co 
Resumen. El objetivo principal del presente trabajo fue diseñar un ambiente virtual marítimo con las características técnicas necesarias para simular ejercicios de navegación con buques virtuales tipo GNL en la bahía de Cartagena. Se desarrolló una herramienta virtual en 3D denominada Terminal Marítimo El Cayao y, posteriormente, se puso a prueba en la ejecución de maniobras en el simulador de puente Transas Ntpro 5000 del Centro de Investigación, Desarrollo e Innovación para Actividades Marítimas de la Escuela Naval (Cidiam). Los resultados permitieron determinar que la herramienta desarrollada es efectiva para la simulación de maniobras con buques virtuales. Se concluye que el uso de este tipo de herramienta permitirá a las tripulaciones de los buques de gran tamaño una mejor comprensión de la complejidad de las maniobras que deben realizar durante su ingreso a la bahía de Cartagena, lo cual contribuye a la mitigación de riesgos y errores humanos durante la navegación.

Palabras clave: buques virtuales tipo GNL; escenario virtual; maniobras; simulación; transporte marítimo.

\begin{abstract}
The main objective of this work was to design a virtual maritime environment with the necessary technical characteristics to simulate navigation exercises using virtual LNG carriers in the Bay of Cartagena. A 3D virtual tool was developed called "Maritime Terminal the Cayao," which was later tested by executing maneuvers on the Naval Academy's Center for Research, Development, and Innovation for Maritime Activities' Transas Ntpro 5000 bridge simulator. The results showed that the developed tool is effective for the simulation of maneuvers using virtual ships. It is concluded that the use of this type of tool will provide large vessel crews a better understanding of the complexity of the maneuvers required during their entry into the Bay of Cartagena, contributing to the mitigation of risks and human errors during navigation.
\end{abstract}

Keywords: LNG type virtual ships; maneuvers; maritime transport; simulation; virtual stage.

Resumo. O principal objetivo deste estudo foi criar um ambiente marítimo virtual com as características técnicas necessárias para simular exercícios de navegação com navios virtuais tipo GNL na Baía de Cartagena. Se desenvolveu uma ferramenta virtual em 3D chamada "Terminal Marítimo El Cayao" e posteriormente testada com a execuçáo de manobras no simulador de ponte Transas NTPRO 5000 do Centro de Pesquisa, Desenvolvimento e Inovação para as Atividades Marítimas da Escola Naval (CIDIAM). Os resultados permitiram determinar que a ferramenta desenvolvida é eficaz para a simulação virtual de manobras com navios virtuais. Conclui-se que o uso deste tipo de ferramenta permitirá que as tripulaçóes de navios de grande porte possam ter uma melhor compreensáo da complexidade das manobras a serem executadas na Baía de Cartagena, contribuindo na mitigaçáo de riscos e erros humanos que se apresentam durante a navegaçấo.

Palavras-chave: cenário virtual; manobras; navios virtuais tipo GNL; simulação; transporte marítimo.

Résumé. L’objectif principal de cette étude était de concevoir un environnement maritime virtuel avec les caractéristiques techniques nécessaires pour simuler des exercices de navigation des navires LNG de type virtuel dans la Baie de Cartagena. S’a développé un outil 3D virtuel appelé "Terminal Marítimo El Cayao ", puis testé en exécutant des manœuvres sur le simulateur de pont Transas NTPRO 5000 du Centre de Recherche, de Développement et de I 'innovation pour les Activités Maritimes de Académie Navale (CIDIAM). Les résultats ont permis de déterminer que l'outil développé est efficace pour les manouvres de simulation virtuelle avec des navires. Il est conclu que l'utilisation de ce type d'outil permettra à des équipages de grands navires une meilleure compréhension de la complexité des manœuvres à effectuer lors de l'entrée à la Baie de Cartagena, contribuant ainsi à l'atténuation des risques et l'erreur humaine tout en naviguant.

Mots-clés: manoeuvres ; navires LNG de type virtuel ; scène virtuelle ; simulation ; transport maritime. 


\section{Introducción}

Las terminales portuarias se caracterizan por ser estructuras básicas que hacen parte de las autopistas marítimas del transporte mundial, por lo cual todas las operaciones y maniobras realizadas en estas deben ser optimizadas de manera continua para lograr la máxima productividad a nivel comercial e industrial. Un ejemplo de ello es la aparición del contenedor en el transporte marítimo, que redujo los tiempos y los costos de manipulación de las cargas, lo cual generó, a su vez, un aumento en el flujo de negocios (Ambrosino, Sciomachen, \& Tanfani, 2004). Esto es importante, ya que más del $80 \%$ del comercio internacional se realiza por vía marítima (Astudillo, 2012).

Son muchas las actividades que se llevan a cabo en los terminales marítimos: la manipulación de mercancías, el depósito y almacenaje de estas, la inspección y control de la mercancía, la consolidación y desconsolidación de cargas y los servicios de apoyo a buques, entre otras. Todas estas labores son vitales para el desarrollo de los intereses marítimos de cualquier país, de allí que sea relevante el uso de estrategias y herramientas orientadas a mejorar la planificación de las operaciones portuarias para que sean más agiles y menos riesgosas (Unctad, 1992). Una de esas herramientas es la simulación marítima, método importante para el entrenamiento (Roza, Voogd, \& Sebalj, 2012).

Los simuladores pueden ser definidos como un dispositivo que duplica las características esenciales de una situación y las ejecuta para que sean operadas por una persona. En el caso de los buques, este sistema incluye el puente de un barco a gran escala, un simulador de timón y una unidad de simulador de posicionamiento dinámico. En actividades de desarrollo portuario, maniobras de buques, mejora de la nave, seguridad y eficiencia, el grado de fidelidad del simulador es clave para el buen desempeño (Vincenzi, Wise, Mouloua, \& Hancock, 2008). Este tipo de montajes tiene la capacidad de proporcionar una plataforma de entrenamiento realista y precisa en actividades de alto riesgo (Suppiah, 2007).

Con base en lo descrito, el trabajo presentado propone el diseño de una herramienta virtual tridimensional denominada Terminal Marítimo El Cayao. Esta herramienta fue desarrollada con el software 3DMAX y Adobe Photoshop, y posteriormente fue integrada al simulador de puente Transas Ntpro 5000 del Cidiam, con software Navi-Trainner 5000, que brinda la posibilidad de alimentar la herramienta con información meteorológica y oceanográfica propia del área de estudio (Transas MIP Ltd., 2013; Emery \& Thomson, 2001). Lo anterior se hizo así con el objetivo de evaluar las características de realismo visual del terminal virtual, con base en la ejecución de maniobras de zarpe y atraque con buques virtuales tipo LNG por parte de oficiales y suboficiales de la Armada Nacional. Tal procedimiento permitió determinar el grado de efectividad de la herramienta al ser utilizada en el proceso de simulación marítima orientada al entrenamiento de las tripulaciones de este tipo de embarcaciones. 


\section{Trabajos rellacionados}

Son muchos los estudios que se han realizado a partir de simuladores, con varios escenarios offshore (Håvold, Nistad, Skiri, \& Odegård, 2015). Además, se han realizado investigaciones de la relación piloto-simulador (Hontvedt, 2015; Kolb, 1984; Schön, 1983; Tuomi-Gröhn \& Engeström, 2003) y se han llevado a cabo estudios acerca del grado de confiabilidad de estos equipos (Dahai, Nikolas, \& Dennis, 2008; Krange, Moen, \& Ludvigsen, 2012). Las simulaciones y modelos están en continua evolución y se utilizan para apoyar el análisis de sistemas, diseño, prueba, evaluación y adquisición de embarcaciones, así como la formación y educación de tripulaciones. En el contexto global, un buen ejemplo de ello es el Australian Maritime College, el cual posee un centro de simulaciones marítimas (Center for Marine Simulations) que se viene utilizando para la investigación de los factores humanos y su incidencia en las operaciones marítimas (Kalnay et al., 1996). La base de datos del simulador incluye la mayoría de los puertos de Australia y Nueva Zelanda, así como áreas de Europa, Malasia e Indonesia. También se ofrece formación regular de simulación para pilotos de organizaciones marítimas como TasPorts, los puertos Newcastle Corporation, Rio Tinto y Port Kembla, además de Napier y Tauranga (Nueva Zelanda).

En el contexto latinoamericano, Chile es uno de los países que está siempre a la vanguardia de capacitar personal en navegación, y cuenta también con instalaciones calificadas de simulación. Prueba de su competencia fue la XXII edición del Curso Internacional de Operaciones en Aguas Antárticas, en el que participaron países como Australia, Colombia, Ecuador, España, Nueva Zelanda, Argentina, Sudáfrica y Chile. Este curso prepara y certifica a oficiales y profesionales para navegar en la zona más austral del mundo, y es reconocido por diversas entidades internacionales y gubernamentales por su excelente nivel académico (Roza et al., 2012). Sin embargo, a pesar de que existe un estado del arte relativamente avanzado en modelación numérica de interacción hielo-embarcación (Lubbad \& Løset, 2011), es poco lo que se ha estudiado en ambientes polares la relación costo-beneficio de la simulación marítima, dada la hostilidad de estos ambientes para la navegación (Comisión Colombiana del Océano [CCO], 2014). Brown y Lutzhoft (2014) proponen un modelo de entrenamiento marítimo en simuladores con condiciones de hielo antártico en el Center for Maritime Simulations del Australian Maritime College, donde tuvieron en cuenta factores como el tipo de embarcación, modelación de hielo, factor humano, clima, hardware, ambiente del área e hidrología.

De los países antes referenciados, es de resaltar el caso de España, ya que ha trabajado en instituciones de educación superior para desarrollar simuladores de entrenamiento en operaciones marítimas, dentro de los cuales se puede citar el Entorno de Simulación de Salvamento Marítimo (Ensisam). Este es un simulador monopuesto orientado a la instrucción de procedimientos en situaciones de emergencia en rescates marítimos. Utiliza un casco de realidad virtual y un guante desarrollado específicamente para la aplicación que permite la interacción con el entorno, lo cual facilita el aprendizaje de protocolos 
propios de este tipo de situaciones. Otro ejemplo de simulador marítimo desarrollado en España es el Entorno de Simulación Tridimensional de Bote de Rescate (Estribor), que consiste en un simulador de bote de rescate que introduce, además del sistema de visualización, una plataforma de movimiento (Tobita, Fukuwa, \& Mori, 2009).

De otro lado, en Brasil la tecnología de simulación viene siendo utilizada para la resolución de problemáticas marítimas, tales como las maniobras en canales de navegación, como es el caso del Canal da Galheta en el puerto de Paranaguá. Allí, se buscó estipular la capacidad del canal por medio de la maniobra de atraque y su grado de seguridad, así mismo, se pretendió calcular el tráfico máximo del canal para determinar la capacidad de movimiento de cargas del sistema portuario, teniendo en cuenta las condiciones ambientales propias del puerto en cuestión (Santos, 2014; Tannuri, 2014). Cabe mencionar que en Argentina también son utilizados los escenarios virtuales para la simulación de distintas maniobras orientadas al entrenamiento de las tripulaciones de distintos tipos de buques de la Armada, los cuales se llevan a cabo en el simulador Melipal, que opera en la Escuela de Oficiales de la Armada Argentina, en la Base Puerto Belgrano, Córdoba (Otheguy, Soriano, Boroni, \& Vénere, 2002; Boroni \& Vénere, 2002).

Los trabajos mencionados se basan en el uso de la tecnología para mejorar la seguridad del tráfico marítimo en distintas partes del mundo, más específicamente, en el uso de ambientes virtuales y simuladores para llevar a cabo entrenamiento especializado de tripulaciones de distintas clases de buques, tanto para personal naval como de la marina mercante. Sin embargo, lo expuesto en el presente artículo es inédito para la región Caribe colombiana, debido a que este tipo de tecnología nunca antes había sido utilizada para el diseño de un escenario marítimo virtual con la capacidad de simular distintos tipos de maniobras en un simulador de puente que incorpore las condiciones oceanográficas y meteorológicas propias de la bahía de Cartagena. Lo anterior representa un aporte relevante en la generación de productos tecnológicos que permitan crear procedimientos pertinentes y de calidad orientados a reforzar los programas de entrenamiento de las tripulaciones de las unidades a flote de la Armada Nacional y el gremio marítimo en general, lo cual promueve la seguridad en el mar y el desarrollo del poder marítimo de la Nación.

\section{Definiciones}

En este apartado se presentan las siguientes definiciones y conceptos, necesarios para situar el trabajo realizado en el contexto naval y marítimo: buques tipo LNG, escenario virtual, simulador de puente, buques virtuales, maniobra, zarpe y atraque.

\section{Buques tipo LNG}

Los buques tipo LNG (liquefied natural gas) o buques gaseros son aquellos buques de gran envergadura construidos para transportar a granel gases licuados. Poseen una capacidad de carga aproximada de entre 30.000 y $266.000 \mathrm{~m}^{3}$ (Romero, 2002). 


\section{Escenario virtual}

Es un espacio dinámico y tridimensional apoyado en las tecnologías de la información, que permite la simulación de situaciones y variables complejas con alto contenido gráfico, táctil y de sonido, lo que brinda una experiencia altamente realista al personal que lo utiliza.

\section{Simulador de puente}

Es un simulador de navegación que representa el puente de un buque mercante o naval. Es considerado como de última tecnología, debido a que proporciona una alta sensación de realidad a la hora de simular maniobras marítimas, pues incorpora características tales como efectos de viento, marea, climatología y corrientes marinas.

\section{Buques virtuales}

Es la representación virtual de los barcos de gran calado que, debido a su tamaño y diseño, son utilizados para realizar navegaciones marítimas de gran importancia.

\section{Maniobra}

La maniobra es un procedimiento o método realizado por cualquier artefacto naval autopropulsado del cual se obtiene un resultado determinante. La maniobra de un buque parte, desde sus bases, de conocer cómo es su evolución dependiendo de las características que este posee hasta tener claramente reconocida la importancia de su correcta operación (avance, círculo evolutivo), el manejo de los elementos que lo conforman (máquinas, timones, cabos) y las habilidades con que cuente el talento humano que lo opera o tripula (experiencia, ojo marinero, entrenamiento). Las maniobras navales se pueden clasificar en dos grandes grupos, generales (o de rutina) y especiales (o tácticas). Dentro de las primeras, se pueden considerar aquellas como zarpe, atraque, fondeo, etc.; y dentro de las segundas se pueden considerar algunas como canal barrido, de engaño, formaciones especiales, aquellas derivadas de cada uno de los tipos de guerra/amenaza, etc. (Castañeda, comunicación personal, 2017)

\section{Atraque}

Maniobra que realizan las embarcaciones para tomar puerto y quedar amarradas a este con el objetivo de realizar operaciones de carga o descarga.

\section{Zarpe}

Es la maniobra opuesta al atraque, y consiste en la acción por la cual el barco suelta sus amarras e inicia su separación del muelle.

\section{Metodología para el diseño de la herramienta virtual Terminal Marítimo El Cayao}

En esta sección se presenta la ruta de diseño utilizada para desarrollar la herramienta virtual Terminal Marítimo El Cayao, la cual consta de los siguientes procedimientos: 1) 
levantamiento de información, 2) diseño de prototipos en 3DMAX, 3) diseño de texturas en Photoshop, 4) aplicación de texturas en los modelos 3D, y 5) finalización del diseño e integración al simulador de puente Transas Ntpro 5000 del Cidiam. En las siguientes subsecciones se describe cada uno de los procedimientos mencionados.

\section{Levantamiento de información}

Se recopiló información referente a los planos de construcción del terminal marítimo, además de registro fotográfico, fílmico y capturas de Google Maps y Google Earth de la zona de estudio. Complementario a esto se obtuvo información meteorológica y oceanográfica de carácter público de la bahía de Cartagena a través del Centro de Investigaciones Oceanográficas e Hidrográficas $(\mathrm{CIOH})$, perteneciente a la Dirección General Marítima (Dimar). Todo lo anterior constituyó la materia prima para el diseño del escenario virtual y la simulación de maniobras con buques virtuales en condiciones muy próximas a la realidad.

\section{Diseño de prototipos en 3DMAX}

Se creó un plano en el software 3DMAX basado en los planos de construcción del terminal marítimo, al que se le colocó como textura imágenes suministradas por la Sociedad Portuaria El Cayao (SPEC). Esto con el fin de desarrollar los prototipos manteniendo la alineación, medidas y distancias reales.

De otro lado, en los prototipos diseńados solo se mantuvieron en el plano los lados visibles desde la perspectiva de la bahía de Cartagena, es decir, los polígonos no visibles desde dicha visual fueron eliminados para minimizar el peso del archivo del escenario virtual terminado. Finalmente, se generó la planerview, que es la línea que delimita cada uno de los prototipos del área diseñada, con el fin de facilitar la inclusión del escenario en el software Navi-Trainner 5000.

\section{Diseño de texturas en Photoshop}

Las texturas fueron generadas con base en los planos de construcción suministrados y complementadas con imágenes del área de estudio, las cuales fueron recortadas y superpuestas sobre los prototipos previamente diseñados, factor que promueve el realismo del escenario. Cabe resaltar que, en el caso de aquellas edificaciones que se ubicaron en un ángulo diferente al frontal con respecto a la bahía de Cartagena, fue utilizada la opción "Sesgar" del submenú "Transformar", incluido en el menú "Imagen de Photoshop", la cual permitió ubicar las texturas de acuerdo con las necesidades. Se desarrollaron dos versiones de las imágenes que hacen parte del escenario virtual (versión día y versión noche) aplicando los efectos de iluminación y oscurecimiento que brinda el software para poder realizar simulaciones de navegación en condiciones matinales, diurnas y nocturnas. Una vez culminado el procedimiento, se generaron archivos de texturas (.BMP), con excepción 
de aquellas imágenes con canales Alfa, que se caracterizan porque presentan transparencias cuando son aplicadas.

\section{Aplicación de texturas en los modelos 3D}

Se asignaron las texturas en la paleta de materiales de 3DMAX, teniendo en cuenta que cada ítem de la paleta al que se le asignara una textura debería tener un valor de cero en la opción "Difuse", con la finalidad de obtener una textura más limpia al momento de ser aplicada a los prototipos. Se orientó cada textura trabajada en cada ítem de la paleta de materiales ( 24 en total). Finalmente, cada prototipo o área de prototipos asignados debe contener todas las texturas obtenidas dentro de una misma captura.

\section{Finalización del diseño e integración al simulador de puente Transas Ntpro 5000}

Una vez finalizados los procedimientos anteriores, se realizó una revisión de los diferentes prototipos diseñados y texturizados que hacen parte del escenario virtual, con el objetivo de minimizar errores en la visualización. Posteriormente, gracias al uso del software Scene Editor, se compilaron todos los prototipos en un solo paquete (.CAB), lo que permitió incorporar el escenario completo al software Navi-Trainner 5000 para ser sometido a pruebas de simulación con buques virtuales tipo LNG en el simulador de puente Transas Ntpro 5000 (figura 1). Se resalta que el software en cuestión proporciona modelos matemáticos que calculan el comportamiento de la embarcación, tanto en el plano como en la vertical, cuando esta se encuentra sometida a fuerzas hidrodinámicas que interactúan con el casco, fuerzas producidas por el oleaje, vientos y corrientes; esto, sin dejar de lado las diversas fuerzas de propulsión y gobierno del buque. Por lo anterior, estos modelos se consideran de alta precisión y flexibles, que operan en seis grados de libertad (Transas, 2013).

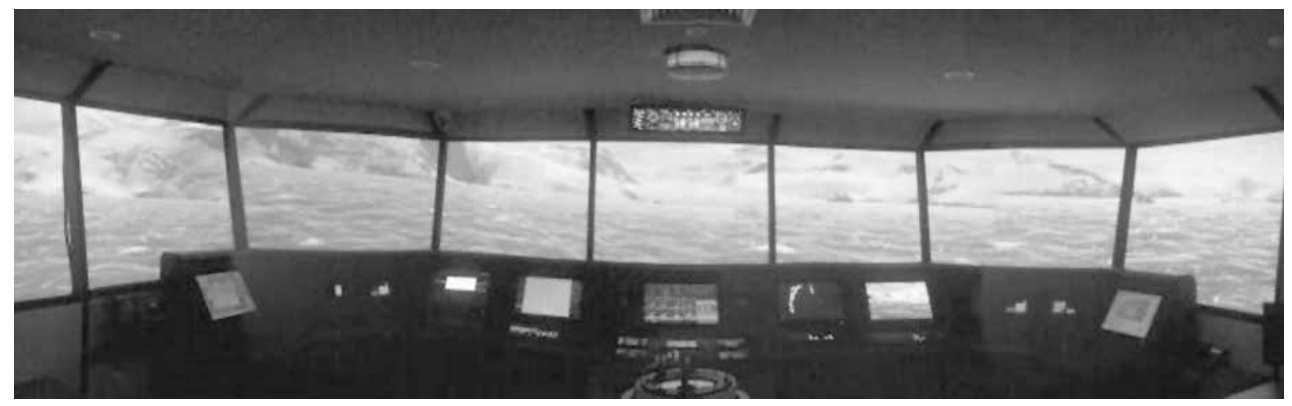

Figura 1. Visualización de un escenario virtual desde el simulador de puente Transas Ntpro 5000 de la Escuela Naval de Cadetes "Almirante Padilla".

Fuente: Cidiam. 


\section{Resulltados y discusión}

En las figuras 2 y 3 se presentan dos perspectivas diferentes del diseño final en 3D del ambiente virtual Terminal Marítimo El Cayao, el cual se desarrolló y perfeccionó en términos de calidad gráfica durante el procedimiento de modelado de prototipos. Lo anterior permitió generar un escenario virtual con características muy cercanas a la realidad en lo concerniente a la infraestructura del puerto, la vegetación aledańa y demás objetos que hacen parte del entorno virtual. Se destaca que se incorporaron condiciones marinas y medioambientales endémicas de la bahía de Cartagena.

Este proceso fue posible gracias a la variada gama de herramientas de diseńo gráfico proporcionadas por el software, así como a la calidad de la información utilizada para el diseño (fotografías y videos). Por lo tanto, se obtuvo como resultado un alto nivel de detalle a la hora de crear, definir y dar forma a cada uno de los entornos que conforman el escenario virtual en cuestión, puesto que se visualizan imágenes de alta resolución que contribuyen a una experiencia de simulación interactiva e inmersiva para los usuarios. Esta calidad posibilita la estandarización de procedimientos que permitirán el entrenamiento de tripulaciones de buques tipo LNG a través de la ejecución de maniobras en tiempo real orientadas a evaluar la toma de decisiones en diferentes circunstancias durante la navegación. Escenarios virtuales con calidad similar han sido diseñados en Latinoamérica por empresas privadas con experticia en el tema, como es el caso del módulo virtual del puerto de Bahía Blanca (Argentina), cuyo fin fue realizar simulaciones con buques virtuales tipo LNG en el área de estudio para determinar el impacto en el tráfico marítimo al momento de realizar su ingreso al puerto (Iribarren et al., 2008). Así mismo, está el caso del escenario virtual de Puerto Cuatreros (Argentina), que fue diseñado para analizar mediante simulación la viabilidad de instalar un terminal de regasificación en dicho punto (Siport21, 2010).

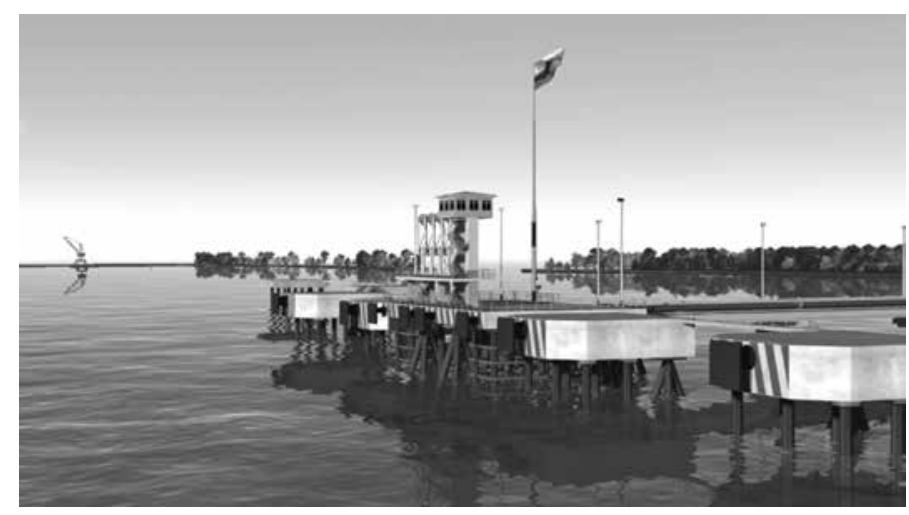

Figura 2. Diseño final del escenario virtual Terminal Marítimo El Cayao, perspectiva lateral. Fuente: Cidiam. 


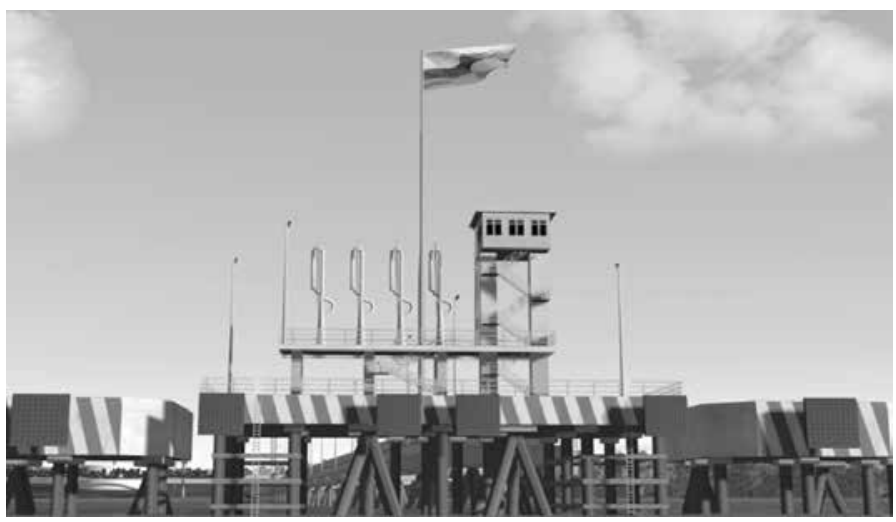

Figura 3. Diseño final del escenario virtual Terminal Marítimo El Cayao, perspectiva frontal. Fuente: Cidiam.

De otro lado, las figuras 4 a 8 ilustran las maniobras con buques virtuales tipo LNG ejecutadas utilizando la herramienta virtual Terminal Marítimo El Cayao, a través del simulador de puente Transas Ntpro 5000 de la Escuela Naval de Cadetes "Almirante Padilla". En cada una de estas imágenes se visualiza una imagen en 3D de los buques virtuales que hacen parte de la galería del simulador interactuando con el ambiente virtual marítimo diseñado en el presente trabajo. La planeación y el desarrollo de cada maniobra estuvieron a cargo de un personal de oficiales y suboficiales de la Armada Nacional en conjunto con pilotos prácticos de la empresa noruega Höegh LNG. Para tal fin se necesitaron alrededor de 20 horas de simulación.

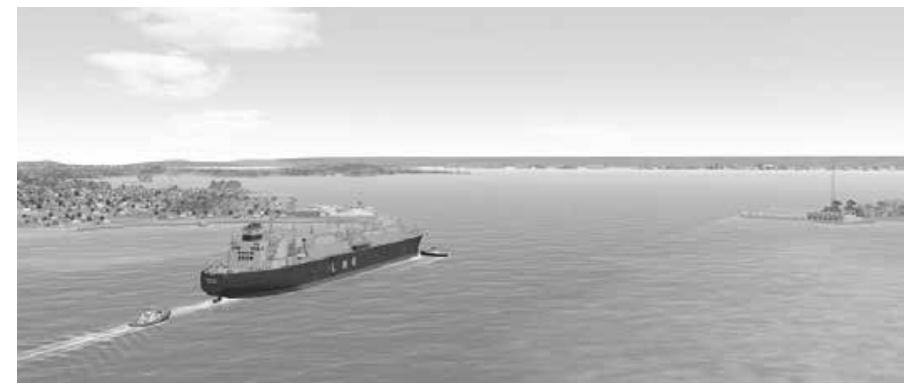

Figura 4. Visualización en 3D de la maniobra de ingreso al canal desde la boya de mar. Fuente: Cidiam.

En la figura 4 se presenta la maniobra denominada "Ingreso al canal desde la boya de mar”, la cual es una maniobra inicial que permite el ingreso del buque LNG a la bahía de Cartagena. En esta operación de acceso a puerto, así como en las demás maniobras ejecutadas en el presente estudio, la herramienta virtual Terminal Marítimo El Cayao permitió incorporar información de factores relevantes para la navegación tales como: corrientes, 
vientos, oleaje, profundidad, entre otras, todo lo cual elevó el nivel de realismo durante el ejercicio. Cabe mencionar que además del buque tipo LNG se utilizaron varios remolcadores virtuales almacenados en la galería de embarcaciones del simulador. Se destaca la capacidad de la herramienta para permitir la interacción exitosa entre distintos tipos de embarcaciones en diversas condiciones medioambientales marinas específicas, de acuerdo con las necesidades de la simulación, lo que contribuyó con el correcto, ágil y fluido desarrollo del ejercicio de navegación planteado.

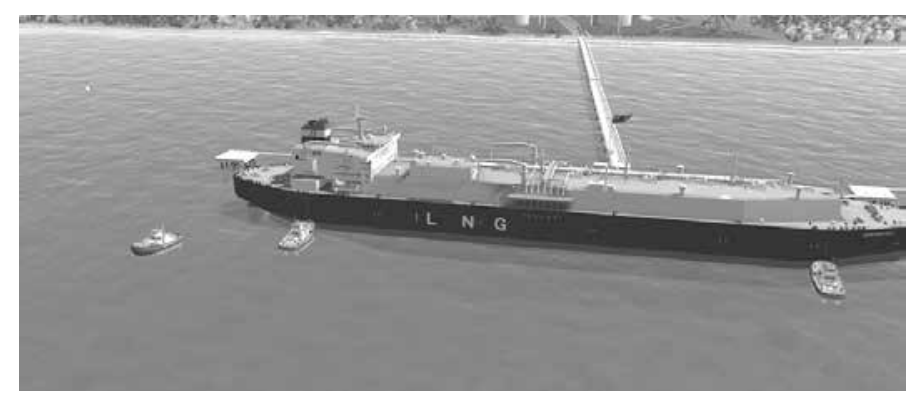

Figura 5. Visualización en 3D de la maniobra de aproximación al muelle.

Fuente: Cidiam.

La figura 5 permite visualizar la ejecución de la maniobra de "Aproximación al muelle", en la cual se observa el acercamiento del buque LNG al Terminal Marítimo El Cayao, con el apoyo de cuatro remolcadores. La calidad visual del ambiente virtual facilitó una condición mínima de gobierno del buque durante la simulación del ejercicio, lo que se tradujo en el control de su velocidad al momento de acercarse al muelle para efectuar el atraque. Dicho control resultó determinante a la hora de lograr una operación exitosa, debido a que la velocidad de aproximación está relacionada directamente con el correcto desplazamiento del buque y la respuesta por parte de sus máquinas. Se enfatiza que la maniobra se realizó a una velocidad inferior a un nudo, por lo cual se requirió asistencia longitudinal de remolcadores.

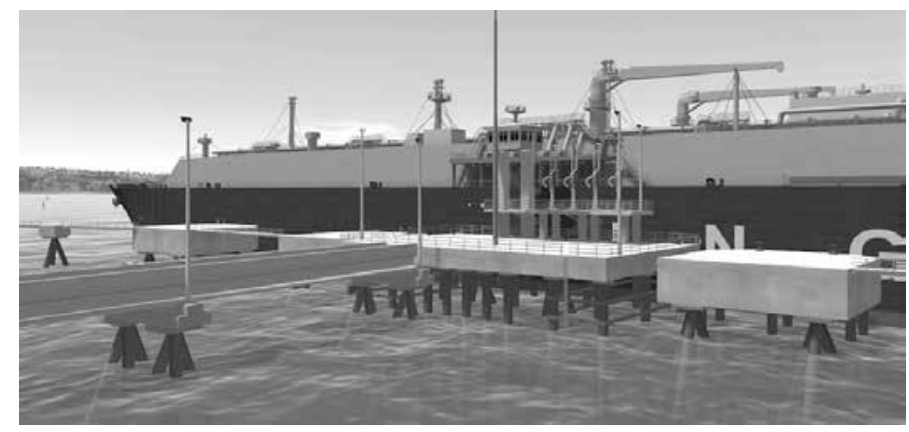

Figura 6. Visualización en 3D de la maniobra de atraque.

Fuente: Cidiam. 
En la figura 6 se observa la maniobra de "Atraque" del buque tipo LNG al Terminal Marítimo El Cayao, la cual inició con la maniobra de "Aproximación al muelle" descrita con antelación, y culminó con el acercamiento total del buque al terminal marítimo. Para tal fin se utilizaron varias velocidades cortas adquiridas por máquina, para evitar el uso prolongado de una alta velocidad que requiera la inversión del propulsor. Por tratarse de un buque de gran envergadura, el control de la velocidad durante el ejercicio se realizó mediante el uso del radar, con referencias a objetos significativos cercanos presentes en el ambiente virtual, lo que facilitó un control total de las distancias, así como minimizar errores procedimentales durante el acercamiento. Cabe resaltar que la maniobra de "Aproximación final" se ejecutó con el buque casi detenido a una eslora del atraque y con el apoyo de cuatro remolcadores. El éxito de la maniobra en gran medida se debió a la fluidez y el alto grado de realismo proporcionado por el escenario virtual diseñado, lo que posibilitó un control total del ejercicio por parte de la tripulación ubicada en el simulador de puente principal y auxiliar.

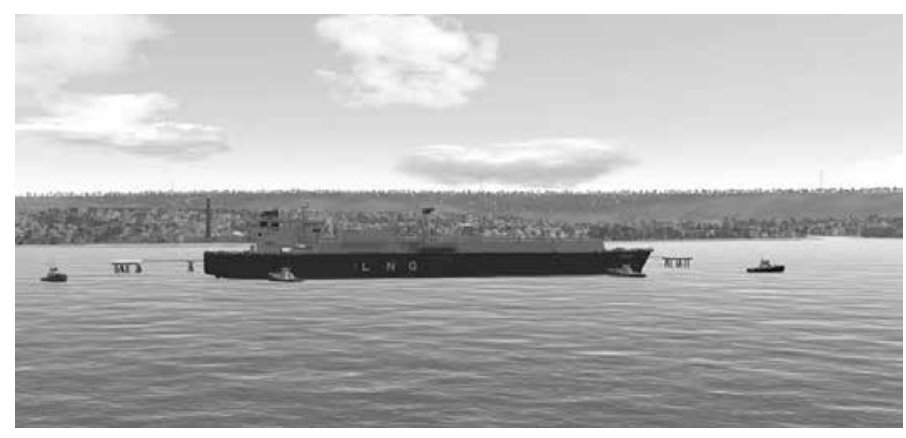

Figura 7. Visualización en 3D de la maniobra de zarpe. Fuente: Cidiam.

Como se puede apreciar en la figura 7 , se visualiza la fase inicial de la maniobra de zarpe de un buque virtual tipo LNG con babor atracado al Terminal Marítimo El Cayao con el apoyo de dos remolcadores. Este ejercicio consistió en la separación gradual del buque del puerto en condiciones de vientos y corrientes mínimas, para lo cual se utilizó solo el esprín de proa con poca velocidad avante y con todo el timón a babor, lo que generó que la popa se separara lo suficientemente del muelle. Después se procedió a ubicar el timón al medio; las velocidades se usaron en la siguiente secuencia: atrás-media-larga, y se culminó con un esprín. Finalmente, al estar bien separado del terminal, se pararon máquinas con timón a estribor y se gobernó la salida avante con baja velocidad.

De acuerdo con las maniobras descritas, la Armada Argentina ha estado realizando ejercicios semejantes de simulación en navegación marítima, ya que adquirieron un simulador de puente denominado Melipal. Este simulador cuenta con herramientas tecnológicas tales como un editor gráfico desarrollado por la Universidad Nacional del Centro de la 
Provincia de Buenos Aires (De Amato, García Bauza, \& Vénere, 2005), cuya finalidad es el diseño de escenarios virtuales enfocados en el entrenamiento de personal civil y militar que hace parte de las tripulaciones de diversos tipos de embarcaciones navales y mercantes (Yang, Wang, \& Chen, 2002). Sin embargo, el editor gráfico en cuestión utiliza una base de datos conformada por una serie de prototipos en $3 \mathrm{D}$ prediseñados (objetos, edificaciones, vegetación, etc.) e imágenes satelitales de áreas de entrenamiento, con el objetivo de diseñar y adaptar escenarios virtuales que posteriormente se utilizarán en las simulaciones (D’Amato, García, Cifuentes, \& Vénere , 2017). A diferencia de esto, en la Escuela Naval de Cadetes "Almirante Padilla", para la generación de ambientes virtuales de simulación se realiza un procedimiento más minucioso, el cual inicia con salidas de campo al área de interés para levantar información fílmica y fotográfica de primera mano, que constituye la base para el diseño de un ambiente virtual con características de realismo idóneas a la hora de simular maniobras de navegación; por lo tanto, todos los objetos y edificaciones que hacen parte del entorno interactivo son diseñados individualmente por el personal del Cidiam.

De otro lado, se resalta que las simulaciones llevadas a cabo en los ambientes virtuales desarrollados por la Armada Argentina presentaron una gran efectividad a la hora de realizar evaluación de áreas de navegación y maniobra, evaluación de límites de distintas operaciones y análisis de estrategias de maniobras (De amato, García Bauza, \& Vénere, 2004). Situación similar a la ilustrada se muestra en el presente artículo, en el cual se muestra que las maniobras marítimas planificadas fueron ejecutadas en su totalidad utilizando la herramienta virtual Terminal Marítimo El Cayao. Esta herramienta fue altamente efectiva a la hora de permitir la organización de los ejercicios de navegación, puesto que posibilitó la adición de distintos tipos de embarcación desde la galería del simulador, así como de objetos virtuales tipo boyas, que cumplen un papel de señalización importante dentro de las operaciones marítimas. También se tuvo en cuenta la manipulación de las condiciones oceanográficas y meteorológicas de acuerdo con las necesidades de las simulaciones en una zona de alto tráfico marítimo como lo es la bahía de Cartagena.

Con base en lo explicado y en lo presentado en las figuras anteriores, se puede afirmar que el enfoque planteado en el presente trabajo permitió crear de manera óptima una herramienta virtual para la navegación marítima altamente interactiva, que posibilitó a los usuarios recrear maniobras con buques virtuales de gran tamaño en condiciones específicas y facilitar, así, la evaluación de toma de decisiones por parte del factor humano directamente implicado en los ejercicios. Cabe recalcar que con este trabajo la Armada Nacional, a través de la Escuela Naval de Cadetes "Almirante Padilla", se convierte en un referente en Colombia en el desarrollo de productos tecnológicos de realidad virtual. A través de estos productos se pretende realizar aportes relevantes en términos de protocolos y procedimientos de seguridad marítima que minimicen el riesgo asociado a errores humanos a la hora de ejecutar maniobras con buques de gran calado en el Caribe colombiano. Lo anterior conlleva la generación de soluciones tangibles a problemáticas existentes 
en el transporte vía marítima, las cuales, gracias a la flexibilidad que brinda la tecnología, se pueden replicar en cualquier entorno marítimo a nivel mundial. De esta forma se contribuye a la capacitación del personal de la Armada Nacional y, en general, de la gente de mar, en pro del fortalecimiento del poder marítimo del país.

\section{Conclusiones}

De los resultados y el análisis presentado se pueden enunciar las siguientes conclusiones sobre el desarrollo de la herramienta: 1) la implementación de tecnologías como el software 3DMAX, Adobe Photoshop, Google Maps y Google Earth en el diseño de escenarios virtuales marítimos es adecuada, debido a que estas brindan las herramientas necesarias para el desarrollo de prototipos de realidad virtual de alta resolución y realismo; 2) la herramienta diseñada contribuye significativamente con el fortalecimiento de la seguridad en el mar, puesto que facilita el análisis y el entendimiento del grado de complejidad que implica el desarrollo de maniobras marítimas con buques de gran envergadura en zonas de alto tráfico marítimo; 3) el escenario virtual proporciona a los usuarios una experiencia altamente inmersiva durante la simulación, complementada por la integración de las condiciones meteorológicas más representativas de la zona de estudio, un alto nivel de detalle gráfico y una interacción fluida con la tripulación que opera desde el simulador de puente; 4) el ambiente virtual desarrollado es efectivo en la simulación de maniobras marítimas con buques virtuales tipo LNG, ya que a través de su uso es posible valorar con un alto grado de exactitud las dificultades de ejecución de las maniobras planteadas.

\section{Agradecimientos}

Los autores agradecen al profesor Jean Roy por su inestimable ayuda durante la redacción de esta comunicación.

\section{Declaración de divullgación}

Los autores declaran que no existe ningún potencial conflicto de interés relacionado con el artículo. Artículo de investigación, asociado al proyecto "Diseño virtual del Terminal Marítimo El Cayao e implementación de prototipos de buques virtuales tipo LNG con capacidad interactiva para simulación de maniobras en puerto y aguas abiertas en un escenario marítimo en 3D de la bahía de Cartagena”, Centro de Investigación, Desarrollo e Innovación para Actividades Marítimas (Cidiam), Facultad de Ciencias Navales y Náuticas, Escuela Naval de Cadetes "Almirante Padilla".

\section{Financiamiento}

Los autores no declaran fuente de financiamiento para la realización de este artículo. 


\section{Sobre los autores}

Cristhian Carmelo Mendoza Pérez es biólogo, Universidad de Córdoba, Montería, Colombia. MSc en Biología, Universidad Federal de Viçosa, Viçosa-Minas Gerais, Brasil. Docente investigador y coordinador del Grupo de Investigación Poseidón, Facultad de Ciencias Navales y Náuticas.

Marena del Rosario Vitola Quintero es ingeniera de sistemas, Fundación Universitaria Tecnológico Comfenalco, Cartagena, Colombia. Especialista en Telecomunicaciones, Universidad Autónoma de Bucaramanga, Bucaramanga, Colombia. MSc en Diseño, Gestión y Dirección de Proyectos, Universidad Internacional Interamericana, San Juan, Puerto Rico. Docente Investigador del Grupo Poseidón y Jefe del Programa de Ciencias Navales, Facultad de Ciencias Navales y Náuticas.

\section{Referencias}

Ambrosino, D., Sciomachen, A., \& Tanfani, E. (2004). Stowing a containership: the master bay plan problem. Transportation Research Part A: Policy and Practice, 38(2), 81-99.

Astudillo, R. (2012). La seguridad marítima en el comercio internacional. Revista de Marina, 57-65. Recuperado de: https://revistamarina.cl/revistas/2012/1/astudillo.pdf

Boroni, G., \& Vénere, M. (2002). Un simulador distribuido para entrenamiento de operarios. Proceedings VIII Congreso Argentino de Ciencias de la Computación. Recuperado de https://goo.gl/XUv4hv.

Brown, P. E., \& Lutzhoft, M. (2014, October). The Ice is right: Antarctic ice modelling in maritime training simulators. Proceedings of the 15th Annual general assembly International Association of Maritime Universities - Innovation in Maritime Education, Training and Research, Australian Maritime College, Launceston, Tasmania. Recuperado de https://goo.gl/W3Saa8.

Castañeda, F. (2017). Comunicación personal, 3 de abril.

Comisión Colombiana del Océano (CCO). (2014) Comité Técnico Nacional de Asuntos Antárticos. Agenda Cientifica Antártica de Colombia 2014-2035. Bogotá, D. C.: Comisión Nacional del Océano.

Dahai, L., Nikolas, M., \& Dennis, V. (2008). Simulation fidelity. In D. A. Vincenzi, J. A. Wise, M. Mouloua, \& P. A. Hancock (Eds.), Factores humanos en simulación y entrenamiento (pp. 61-73). Boca Raton: CRC Press.

De Amato, J., García Bauza, C., \& Vénere, M. (2004). Simulación del entorno de una embarcación pesquera. 33. as Jornadas Argentinas de Informática e Investigación Operativa, JAIIO 2004, Córdoba.

De Amato, J., García Bauza, C., \& Vénere, M. (2005). Editor de escenarios para aplicaciones de realidad virtual. 34. Jornadas Argentinas de Informática e Investigación Operativa, JAIIO 2005, Córdoba.

De Amato, J. P., García, C., Cifuentes, V., \& Vénere , M. (15 de 08 de 2017). Universidad Tecnológica Nacional. Obtenido de www.http://utn.edu.ar/: http://jidis.frc.utn.edu.ar/papers/8cf012187596558f1101bd409792.pdf

Emery, W. J., \& Thomson, R. E. (2001). Data analysis methods in physical oceanography (2nd ed.). New York: Elsevier Sci.

Håvold J., Nistad, S., Skiri, A., \& Odegård A. (2015). The human factor and simulator training for offshore anchor handling operators. Safety Science, 75, 136-145. doi: 10.1016/j.ssci.2015.02.001.

Hontvedt, M. (2015). Professional vision in simulated environments - Examining professional maritime pilots' performance of work tasks in a full-mission ship simulator. Learning, Culture and Social Interaction, 7, 71-84. doi: 10.1016/j.lcsi.2015.07.003. 
Iribarren, J. R., Cal, C., López, L., Ibáńez, A. C., Weis, C., Zickermann, J., \& Murguialday, J. (2008). Sistema innovador para el suministro de LNG en la Terminal Bahía Blanca de Argentina. Recuperado de https://goo.gl/qxBuE9.

Kalnay, E., Kanamitsu, M., Kistler, R., Collins, W., Deaven, D., Gandin, L., ... Denis, J. (1996). The NCEP/NCAR 40-year reanalysis project. Bulletin American Meteorological Society, 77, 437-470. doi: 10.1175/1520-0477(1996)077<0437:TNYRP>2.0.CO;2.

Kolb, D. A. (1984). Experiential learning: Experience as the source of learning and development. New Jersey: Prentice-Hall.

Krange, I., Moen, A., \& Ludvigsen, S. (2012). Computer-based 3-D simulation: A study of communication practices in a trauma team performing patient examination and diagnostic work. Instructional Science, 40(5), 829-847.

Lubbad, R., \& Løset, S. (2011). A numerical model for real-time simulation of ship-ice interaction. Cold Regions Science and Technology, 65(2), 111-127. doi: 10.1016/j.coldregions.2010.09.004.

Otheguy, I., Soriano, M., Boroni, G., \& Vénere M. (2002). Simulación en tiempo real de un radar de barrido horizontal. Mecánica Computacional Vol XXI. (pp. 1203-1212). Recuperado de: https:// www.researchgate.net/publication/228681230_Simulacion_en_tiempo_real_de_un_radar_de_ barrido_horizontal

Romero, R. (2002). El transporte marítimo: introducción a la gestión del transporte maritimo (1. ${ }^{\mathrm{a}}$ ed.). Barcelona (España): Marge Books.

Roza, M., Voogd, J., \& Sebalj, D. (2012). The generic methodology for verification and validation to support acceptance of models, simulations and data. The Journal of Defense Modeling and Simulation: Applications, Methodology, Technology, 10(4), 347-365.

Santos, S. (2014). Aspectos da navegação interior. Florianópolis: Labtrans/UFSC.

Schön, D. A. (1983). El profesional reflexivo. Cómo piensan los profesionales cuando actúan. San Francisco: Jossey-Bass.

Siport21. (2010). Análisis de viabilidad de la instalación de una terminal de regasificación en Puerto Cuatreros. Recuperado de http://es.siport21.com/projects/analisis-de-viabilidad-de-la-instalacion-de-una-terminal-de-regasificacion-en-puerto-cuatreros/.

Suppiah, R. (2007). Bridging the gap between theory and practice in the maritime environment: implications for Educators. Maritime Studies, 153, 17-20.

Tannuri, E. (2014). Modelagem matemática de manobras e validação de simuladores. Recuperado de https:// goo.gl/hWGGhS.

Tobita, J., Fukuwa, N., \& Mori, M. (2009). Integrated disaster simulator using WebGIS and its application to community disaster mitigation activities. Journal of Natural Disaster Science, 30(2), 71-82. doi: 10.2328/jnds.30.71.

Transas MIP Ltd. (2013). Navy Trainer 5000. Version 5.3.

Tuomi-Gröhn, T., \& Engeström, Y. (2003). Between school and work: New perspectives on transfer and boundary-crossing. Amsterdam: Pergamon Press.

Unctad. (1992). Development and inprovement of ports: The principles of modern port management and organization. Ginebra: United Nations Conference on Trade and Development, Trade and Development Board, Committee on Shipping, Ad hoc Intergovernmental Group of Port Experts.

Vincenzi, D., Wise, J., Mouloua, M., \& Hancock, P. (2008). Human factors in simulation and training. Boca Raton, CA: CRC Press.

Yang, Y. J., Wang, X. S., \& Chen, J. X. (2002). Rendering avatars in virtual reality: Integrating a 3D model with 2D images. Computing in Science \& Engineering, 4(2), 86-91. doi: 10.1109/5992.976440. 